\title{
Evaluation of hearing deficit in patients with oral submucous fibrosis
}

\author{
Parvathi Devi, Ipsa Singh, Ravindra Setru, Kuber Tyagi, \\ Dharamveer Singh, and Bellona Thiyam
}

Department of Oral Medicine and Radiology, Teerthanker Mahaveer Dental College
and Research Centre, Moradabad, India

(Received September 5, 2014; Accepted March 27, 2015)

\begin{abstract}
Oral submucous fibrosis (OSMF) is a well recognized premalignant condition predominantly affecting the oral cavity, but sometimes extending to the pharynx, esophagus or even the larynx. OSMF may lead to stiffness of the oral cavity, resulting in trismus and inability to eat, difficulty with speech or swallowing, pain in the throat and ears, and a relative loss of auditory acuity. To evaluate the hearing deficit in patients with OSMF, we examined a study group comprising 40 patients, and also 10 age- and sex-matched healthy subjects as controls. All of the subjects were evaluated for hearing loss by pure tone audiometry. The OSMF group showed a significant degree of hearing loss relative to the control group. OSMF in advanced stages was significantly associated with mild conductive hearing loss $(P<0.01)$. The present study revealed a significant association between OSMF and hearing deficit. Involvement of the palatal muscles with OSMF may decrease the patency of the Eustachian tube, leading to conductive hearing loss. Therefore, all patients with OSMF should be evaluated for hearing deficit and advised about appropriate treatment.
\end{abstract}

(J Oral Sci 57, 109-113, 2015)

Keywords: oral submucous fibrosis; palatal muscles; pure tone audiometry; conductive hearing loss.

Correspondence to Dr. Ipsa Singh, Department of Oral Medicine and Radiology, Teerthanker Mahaveer Dental College and Research Center, NH 24, Bagarpur, Delhi Road, Moradabad 244001, India

E-mail: ipsacoolone@gmail.com

doi.org/10.2334/josnusd.57.109

DN/JST.JSTAGE/josnusd/57.109

\section{Introduction}

Oral submucous fibrosis (OSMF) is a chronic progressive and irreversible disease affecting the oral, oropharyngeal and sometimes the esophageal mucous membrane. OSMF causes changes similar to those of systemic sclerosis (scleroderma), but limited to oral tissues (1). A condition resembling OSMF was described as early as $600 \mathrm{BC}$ by Sushruta, and it was named 'vidari' having features of progressive narrowing of the mouth, depigmentation of the oral mucosa and pain on taking food (2). In 1952, Schwartz described five Indian women from Kenya with a condition of the oral mucosa including the palate and pillars of the fauces, which he called "atrophia idiopathica (tropica) mucosae oris" (3). Subsequently, in 1953, Joshi coined the term OSMF for the condition (2). Most of the large-scale studies conducted previously have been reported from India, Pakistan, South Africa, Taiwan, and South East Asian countries. It is a well recognized potentially premalignant condition that predominantly affects the oral cavity, but may extend to the pharynx, esophagus and even to the larynx. It is characterized by a juxta-epithelial inflammatory reaction followed by fibroelastic changes in the lamina propria with epithelial atrophy, causing stiffness of the oral cavity resulting in trismus and inability to eat (4).

OSMF also leads to difficulty in speech and swallowing, pain in the throat and ears, and a relative loss of auditory acuity (5). Many studies of OSMF have demonstrated progressive degeneration of the underlying musculature, causing impairment of function. Palatal involvement is seen in more than $50 \%$ of patients with fibrosis evident in the faucial pillars (6). Involvement of 
Table 1 Qualitative hearing loss

\begin{tabular}{llll}
\hline Type of hearing impairment & Air conduction & Bone conduction & A-B gap \\
\hline Normal & Normal & Normal & Minimal \\
Conductive & Decreased & Normal & More than 20 \\
Sensorineural & Decreased & Decreased & More than 15 \\
Mixed & Decreased & Decreased & More than 20 \\
\hline
\end{tabular}

the palatal and paratubal muscles (levator veli palatini, tensor veli palatini, tensor tympani and salpingopharyngeus), which regulate the patency and function of the pharyngeal orifice, results in impairment of eustachian tube function and patency (7), leading to pain in the ear along with conductive loss of hearing (6). Despite extensive studies of the etiopathogenesis, clinical presentation and management of OSMF, only a few studies have investigated the impairment of eustachian tube patency and function in affected patients. The aim of the present study was to evaluate the hearing deficit in patients with OSMF.

\section{Materials and Methods}

The present study was carried out at the Department of Oral Medicine and Radiology to evaluate hearing deficits in patients with OSMF. The study protocol was approved by the Institutional Ethics Committee (TMDCRC/Acad/ OMR, 2012), and after obtaining written informed consent, the clinical profiles of the patients were by taken from a thorough case history and clinical examination. The patients had been referred to an ENT specialist for clinical examination of the ear in order to exclude ear infections and any other abnormalities. Patients diagnosed as having OSMF were included in the study, and those with pathology of the middle ear, e.g., tympanic membrane perforation, cholesteatoma, or previous surgery were excluded. The study group comprised 40 subjects, who were classified into four groups of 10 patients each: Group A (interincisal distance $\geq 35 \mathrm{~mm}$ ), Group B (30-35 mm), Group C (20-30 mm) and Group D $(<20 \mathrm{~mm})$ using the Clinical Classification of Lai (8). The control group comprised 10 healthy subjects. Audiological assessment was done using ALP 2100 clinical audiometer (ALPS International, New Delhi, India). Both the right and left ears of all the subjects were evaluated for air conduction hearing loss and bone conductive hearing loss.

\section{Audiometry procedure}

In pure tone audiometry, pure tone is delivered to the ear through headphones for air conduction and by a bone vibrator for bone conduction. Hearing level in decibels above the normal threshold is plotted. The frequency tested usually ranged from 250 to $8,000 \mathrm{~Hz}$. The pure tone average is the average of the hearing threshold levels at 500,1,000, 2,000 Hz only (5). Qualitative hearing loss was recorded as shown in Table 1 (9, www. bhojvirtualuniversity.com).

The deafness can be graded into several categories by the air conduction threshold (5), i.e.:

1. 10-15 dB - Normal hearing

2. 16-25 dB - Minimal hearing loss

3. 26-40 dB - Mild

4. 41-55 dB - Moderate

5. 56-70 dB - Moderate to severe

6. 71-90 dB - Severe

7. $>90 \mathrm{~dB}$ - Profound deafness

Hearing impairment or deafness can be classified qualitatively into normal, conductive, mixed or sensorineural. The overall extent and type of hearing impairment was described in single terminology by using the quantitative grade and qualitative type of hearing impairment together.

\section{Results}

Statistical analysis was performed using SPSS version 19 and Epi. Info. version 3 for Microsoft Windows. The tests used were paired $t$ test and Pearson's chi-squared to find the association between the grade of OSMF and hearing deficit. The mean age of the subjects, including controls, was $30.82 \pm 7.72$ years. Mean quantitative hearing loss in decibels in the right ear was $22.06 \pm 9.19$ $\mathrm{dB}$, and that in the left ear was $22.06 \pm 7.09 \mathrm{~dB}$ (Fig. 1). Among the 80 ears in the 40 OSMF cases, hearing was normal in 45 ears $(56 \%)$, mild conductive hearing loss was found in 31 ears (39\%), mild sensorineural hearing loss in 1 ear (1\%) and moderate sensorineural hearing loss was present in 3 ears (4\%). Among the 20 ears in the 10 controls, hearing was normal in 19 ears (95\%), and mild conductive hearing loss was found in 1 ear (5\%) (Figs. 2, 3). Paired $t$ test was applied to assess the associations among the various OSMF groups, and quantitative hearing loss in the right and left ear, respectively. No significant difference in mean quantitative hearing loss was observed for OSMF groups A and B, whereas a significant difference of mean quantitative hearing loss was evident in OSMF groups $\mathrm{C}$ and group $\mathrm{D}$, relative to 


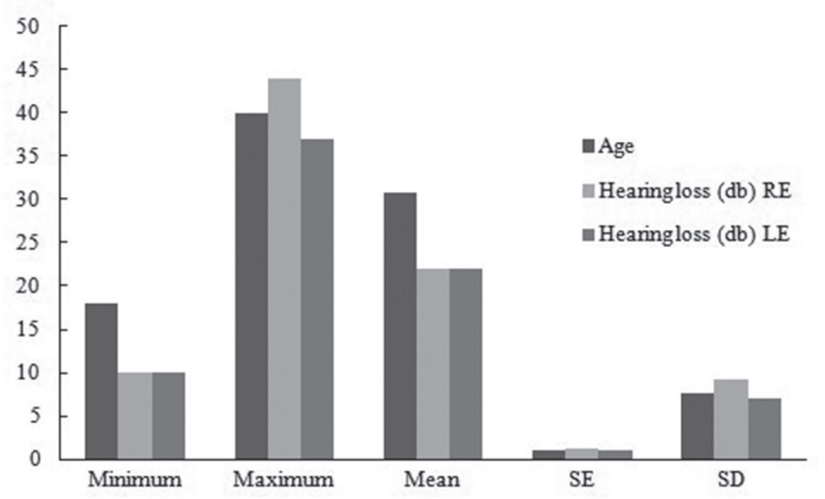

Fig. 1 Descriptive statistics of the study subjects.

Forty subjects with OSMF comprised the study group and 10 healthy subjects comprised the control group, the mean age of the subjects overall being $30.82 \pm 7.72$ years. Mean quantitative hearing loss in decibels in the right ear was found to be $22.06 \pm$ $9.19 \mathrm{~dB}$, and for left ear $22.06 \pm 7.09 \mathrm{~dB}$.

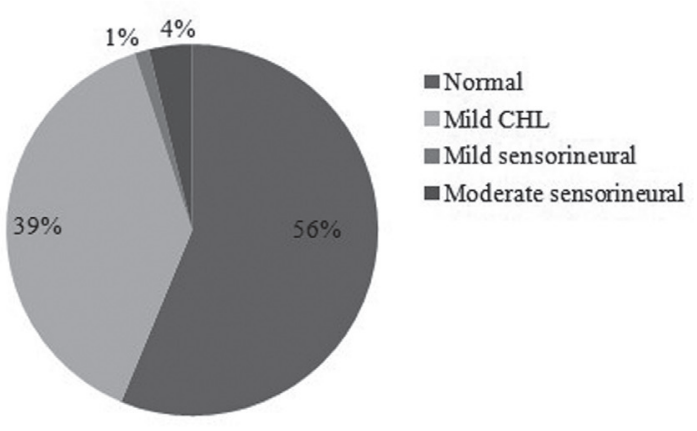

Fig. 2 Overall percentage of qualitative hearing loss in OSMF subjects.

Out of 80 ears in 40 OSMF cases, hearing was found to be normal in 45 ears (56\%), mild conductive hearing loss was found in 31 ears (39\%), mild sensorineural hearing loss was found in 1 ear $(1 \%)$, and moderate sensorineural hearing loss was present in 3 ears $(4 \%)$.

the control group (Table 2). Pearson's chi-squared test was applied to assess the associations among the various OSMF groups and qualitative grades of hearing loss in the right ear and left ear, respectively. With a chi-squared value of 37.8 and 35.4 for the right and left ear, respectively, at eight degrees of freedom, the null hypothesis of no difference was rejected at $P<0.001$. Hence there is a strong association between OSMF and hearing loss in both ears. OSMF Group D was significantly associated with mild conductive hearing loss in both ears (Table 3). Group D was significantly associated with mild conductive hearing loss, and normal hearing was significantly associated with the control group as compared to any other group in both the right and left ears $(P<0.001$, being highly significant) (Fig. 4).

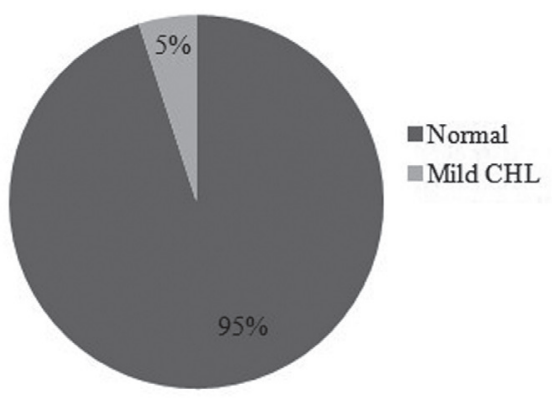

Fig. 3 Overall percentage of qualitative hearing loss in the control group.

Out of 20 ears in 10 control cases, hearing was found to be normal in 19 ears (95\%) and mild conductive hearing loss was found in 1 ear $(5 \%)$.

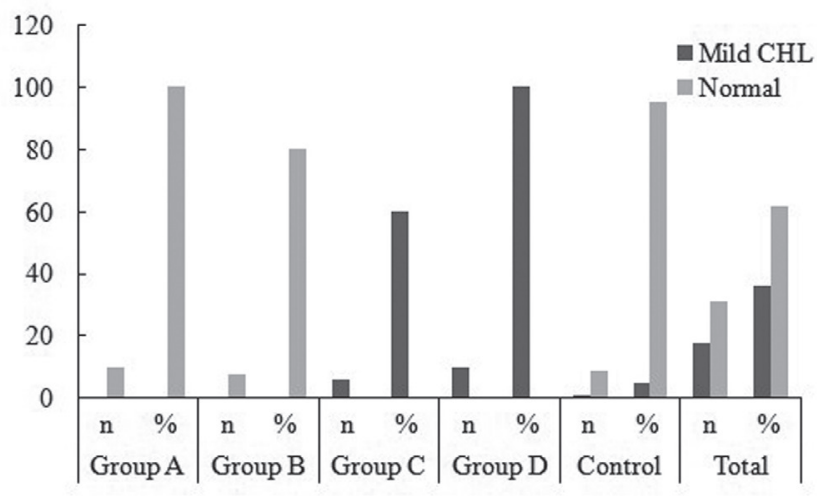

Fig. 4 Qualitative hearing loss among the various OSMF groups. OSMF Group D was significantly associated with mild conductive hearing loss, and normal hearing was significantly associated with the control group as compared to any other group in both the right and left ears $(P<0.001$, being statistically highly significant).

\section{Discussion}

Oral submucous fibrosis (OSMF) is a well-established precancerous condition affecting the oral mucosa (2). Pindborg and Sirsat in 1966 (1) defined OSMF as "an insidious chronic disease affecting any part of the oral cavity and sometimes the pharynx. Although occasionally preceded by and/or associated with vesicle formation, it is always associated with juxta-epithelial inflammatory reaction followed by a fibroelastic change of the lamina propria with epithelial atrophy leading to stiffness of the oral mucosa and causing trismus and inability to eat".

Early symptoms of OSMF include a burning sensation in the mouth, appearance of vesicles, and recurrent generalized inflammation of the mucosa. As the disease 
Table 2 Mean quantitative hearing loss in decibels $(\mathrm{dB})$ in both ears among the various OSMF study groups and the controls

\begin{tabular}{llccccc}
\hline OSMF group & \multicolumn{1}{c}{ Parameter } & $n$ & Mean & SD & SEM & Significance \\
\hline Group A & Hearing loss (db) RE & 10 & 16.30 & 4.373 & 1.383 & $P=0.464 \mathrm{NS}$ \\
& Hearing loss (db) LE & 10 & 17.70 & 3.945 & 1.248 & $P=0.714 \mathrm{NS}$ \\
Group B & Hearing loss (db) RE & 10 & 18.60 & 9.383 & 2.967 & $P=0.811 \mathrm{NS}$ \\
& Hearing loss (db) LE & 10 & 17.70 & 7.454 & 2.357 & $P=0.802 \mathrm{NS}$ \\
Group C & Hearing loss (db) RE & 10 & 24.40 & 9.359 & 2.960 & $P<0.05$ \\
& Hearing loss (db) LE & 10 & 26.30 & 3.889 & 1.230 & $P<0.01$ \\
Group D & Hearing loss (db) RE & 10 & 33.20 & 5.514 & 1.744 & $P<0.01$ \\
& Hearing loss (db) LE & 10 & 30.20 & 4.104 & 1.298 & $P<0.01$ \\
Control group & Hearing loss (db) & 10 & 18.40 & 4.452 & 1.408 & $P=0.464 \mathrm{NS}$ \\
\hline \multicolumn{7}{l}{ RE = right ear; LE $=$ left ear; NS = non significant $P>0.05 ;$ OSMF = Oral submucous fibrosis }
\end{tabular}

Table 3 Pearson chi-squared test and likelihood ratio for cross tabulation-OSMF Group hearing loss (qualitative) in the right ear and left ear

\begin{tabular}{lcccccc}
\hline & \multicolumn{3}{c}{ Right ear } & \multicolumn{2}{c}{ Left ear } \\
\cline { 2 - 6 } & Value & df & Significance & Value & df & Significance \\
\hline Pearson Chi-Square & $37.857^{\mathrm{a}}$ & 8 & $P<0.001$ & $35.430^{\mathrm{a}}$ & 8 & $P<0.001$ \\
Likelihood Ratio & 48.077 & 8 & $P<0.001$ & 41.499 & 8 & $P<0.001$ \\
\hline $\mathrm{df}=$ degrees of freedom, $P<0.001=$ Statistically highly significant. Chi-squared value 37.8 and 35.4 for the right and left \\
ear, respectively, at eight degrees of freedom, the null hypothesis of no difference being rejected at $P<0.001$. Hence there \\
is a strong association between OSMF groups and hearing loss in both ears. OSMF Group D is significantly associated \\
with mild hearing loss CHL in both ears.
\end{tabular}

progresses, there is evident blanching along with fibrosis of the oral mucosa. With progressive fibrosis there is stiffening of areas of the mucosa, leading to difficulty with mouth opening, speaking, and swallowing, as well as taste alteration. Involvement of the nasopharynx may cause pain in the ear and nasal twang (4).

The normal hearing mechanism involves entrance of sound waves into the auditory canal, which then strike the eardrum, causing it to vibrate. The sound waves are amplified by passing from a relatively large area (the eardrum) through the ossicles to a relatively small opening leading to the inner ear. The alternating changes in pressure agitate the basilar membrane on which the organ of Corti rests, moving the hair cells. This movement stimulates the sensory hair cells to send impulses along the auditory nerve to the brain. A small proportion of normal hearing involves direct transmission of sound waves to the inner ear via the bones of the skull, i.e., the auditory canal and the middle ear are bypassed. This kind of hearing is referred to as bone conduction. The Eustachian tube is approximately $3 \mathrm{~mm}$ in diameter (less than $1 / 10$ inch). Cartilage provides the supporting structure for the first two-thirds of the eustachian tube, whereas the last third (closest to the middle ear space) is supported by bone (5). Traditionally, four muscles are commonly cited as being associated with the Eustachian tube: the tensor veli palatini, levator veli palatini, salpingopharyngeus, and tensor tympani. At one time or another, each has been directly or indirectly implicated in tubal function. Usually the eustachian tube is closed, but it opens during actions such as swallowing, yawning, or sneezing, thereby permitting the equalization of middle ear and atmospheric pressures (9). The patency and ventilatory function of the eustachian tube may be impaired if any of these muscles is affected by OSMF (5).

In the present study, pure tone audiometry of 80 ears in 40 OSMF patients revealed that hearing was normal in $56 \%$, mild conductive hearing loss was present in $39 \%$, mild sensorineural hearing loss was present in $4 \%$, and moderate sensorineural hearing loss was evident in $3 \%$. Out of 20 ears in the control group, hearing was found to be normal in 19 ears (95\%), and mild conductive hearing loss was found in 1 ear (5\%). These results were in accordance with the study conducted by Gupta et al. (10), where hearing was found to be normal in $79.2 \%$ of ears, mild to moderate conductive hearing loss was evident in $18.0 \%$, and sensory neural hearing loss was evident in $2.8 \%$. Shah et al. (11) reported that out of 54 ears in their OSMF group, hearing was normal in 67\%, mild sensorineural hearing loss was found in $22 \%$, and moderate mixed hearing loss was present in $11 \%$.

In the present study there was no significant difference in mean hearing loss for OSMF groups A and B, whereas a significant difference in mean hearing loss was observed in OSMF groups $\mathrm{C}$ and group $\mathrm{D}$ as compared with the control group. Group D was significantly associated 
with mild conductive hearing loss, and normal hearing was significantly associated with the control group as compared with any other group in both the right and left ears $(P<0.01$; highly significant). These results were in accordance with those of Chaudhary et al. (5).

Dysfunction of the eustachian tube in OSMF may be due to fibrosis of the palatal muscles, leading to loss of conductive hearing. el Labban and Caniff compared ultrastructural changes in the muscle fibers of normal individuals and OSMF patients, and found severe degenerative changes in a high proportion OSMF patients (12). Gupta et al. (6) reported degenerative changes in the palatal/paratubal muscles in the form of loss of crossstriations in $13.2 \%$ of cases, edematous muscle fibers in $9.4 \%$, and atrophy in $9.4 \%$. That study confirmed that extension of fibrosis into the nasopharynx, involving the pharyngeal orifice of the eustachian tube and changes in the muscles, affected the function of the eustachian tube. Rooban et al. (13) conducted a light microscopic study of fibrosis involving the muscle in OSMF and reported that fibrosis extended into the superficial (40\%) and deeper (10\%) regions without visible changes in the muscle bands. In other OSMF cases, thin collagen fibers showing a broken sarcolemma were present. Furthermore, fibrosis with hyalinization was also seen extending into the muscle band zone, resulting in atrophy of the muscle, whereas in $26.67 \%$ of cases the muscle band area was replaced by fibrous tissue. Sumathi et al. (14) conducted a study to evaluate the ultrastructural features of muscle tissue in the moderate and advanced stages of OSMF in 80 cases and found various changes in a high proportion of muscle fibers, including irregularity of the fiber surfaces, sarcolemmal folding, reduplication of the basement membrane, loss and alteration of myofilaments, and hypercontraction of myofibrils, all of which were suggestive of muscle atrophy and necrosis.

Thus, our present findings suggest that conductive hearing loss is evident in the advanced stages of OSMF because of alterations in the patency of the eustachian tube, which may occur due to progressive fibrosis of the palatal muscles, being directly proportional to the stage of the disease. However, further studies of OSMF at the advanced stage and a larger sample size will be required to further validate these findings.

We found a significant correlation between OSMF and the degree of hearing deficit, especially at the advanced stages, mainly in groups $\mathrm{C}$ and $\mathrm{D}$, which can be correlated with the degree of fibrosis of the palatal muscles, thus reducing the patency of the eustachian tube. As fibrosis of the oropharynx leads to altered perception of sound, all patients with OSMF should be evaluated for hearing deficit. Therefore, the protocol for managing OSMF patients should include ENT consultation and treatment for hearing deficit in order to increase the success rate of treatment.

\section{References}

1. Afroz N, Hasan SA, Naseem S (2006) Oral submucous fibrosis: a distressing disease with malignant potential. Indian J Community Med 31, 270-271.

2. Shevale VV, Kalra RD, Shevale VV, Shringarpure MD (2012) Management of oral sub-mucous fibrosis: a review. Indian J Dent Sci 4, 107-114.

3. Rajendran R (1994) Oral submucous fibrosis: etiology, pathogenesis, and future research. Bull World Health Organ 72, 985-996.

4. Arakeri G, Brennan PA (2013) Oral submucous fibrosis: an overview of the aetiology, pathogenesis, classification, and principles of management. Br J Oral Maxillofac Surg 51, 587-593.

5. Chaudhary MS, Mohite DP, Gupta R, Patil S, Gosavi S, Gawande M et al. (2013) Evaluation of hearing efficiency in patients with oral submucous fibrosis. Otolaryngology, doi: 10.4172/2161-119X.1000143.

6. Gupta SC, Khanna S, Singh M, Singh PA (2000) Histological changes to palatal and paratubal muscles in oral submucous fibrosis. J Laryngol Otol 114, 947-950.

7. Misra SP, Misra V, Dwivedi M, Gupta SC (1998) Oesophageal subepithelial fibrosis: an extension of oral submucosal fibrosis. Postgrad Med J 74, 733-736.

8. More CB, Gupta S, Joshi J, Varma SN (2012) Classification system for oral submucous fibrosis. J Indian Aca Oral Med Radiol 24, 24-29.

9. Ramírez LM, Ballesteros LE, Sandoval GP (2007) Tensor tympani muscle: strange chewing muscle. Med Oral Patol Oral Cir Bucal 12, E96-100.

10. Gupta SC, Singh M, Khanna S, Jain S (2004) Oral submucous fibrosis with its possible effect on eustachian tube functions: a tympanometric study. Indian J Otolaryngol Head Neck Surg 56, 183-185.

11. Shah M, Katarkar A, Shah P, Alam N, Modh D (2011) Tympanometric study of eustachian tube function in oral submucous fibrosis. Indian J Otology 17, 80-82.

12. el-Labban NG, Canniff JP (1985) Ultrastructural findings of muscle degeneration in oral submucous fibrosis. J Oral Pathol 14, 709-717.

13 Rooban T, Saraswathi TR, Al Zainab FH, Devi U, Eligabeth J, Ranganathan K (2005) A light microscopic study of fibrosis involving muscle in oral submucous fibrosis. Indian J Dent Res 16, 131-134.

14. Sumathi MK, Balaji N, Malathi N (2012) A prospective transmission electron microscopic study of muscle status in oral submucous fibrosis along with retrospective analysis of 80 cases of oral submucous fibrosis. J Oral Maxillofac Pathol $16,318-324$. 